\title{
Passing VBR in Mobile Ad Hoc Networks - for effective live video Streaming
}

\author{
V. Saravanan \\ Asst.Professor in Computer Applications, \\ Hindusthan College of arts and Science, \\ Coimbatore, India
}

\author{
Dr.C.Chandrasekar \\ Associate Professor, \\ Dept.of Computer Science, \\ Periyar University, Salem, India
}

\begin{abstract}
Mobile ad hoc networks (often referred to as MANETs) consist of wireless hosts that communicate with each other in the absence of a fixed infrastructure. This technique can be used effectively in disaster management, intellectual conference and also in the battlefield environments. It has the significant attention in the recent years. This research paper depicts the remuneration of using suggestion tracking for selecting energy-conserving routes in delay-tolerant applications and it sends Variable Bit Rate delivery. The previous investigation set up from earlier period surveillance that delay can be traded for energy efficiency in selecting a path. The Prior objective is to find an experiential upper bound on the energy savings by assuming that each node accurately knows or predicts its future path. It examines the effect of varying the amount of future information on routing. Such a bound may prove useful in deciding how far to look in advance, and thus how much convolution to provide in mobility tracking.
\end{abstract}

Keywords-Variable Bit Rate; Mobile Ad Hoc; Machine Learning.

\section{INTRODUCTION}

Mobile ad hoc networks are a set of peer-to-peer reconfigurable networks. In general, any pair of associated nodes may communicate with one another, using transitional nodes to store and forward frames. The annoyance of having a flat hierarchy is that the capacity of the network is expended forwarding other nodes' data. In this paper, we concentrate on Mobile Ad Hoc Networks with a large number of nodes with delay-broadminded applications. Under these conditions, properly delaying forwarding can greatly increase the transport capacity or, equivalently, the lifetime of the network. In addition, it is used in accounting for their complexities. For even greater savings, mobility tracking may be augmented by the following machine learning methods:

- Mechanical classification coordination to avoid channel disputation - passage-tracking

- $\quad$ To keep up connectivity by significant how much energy is absent: Energy-tracking

- To stay away from dead ends with geographic steering: Route Map-structure.

\section{VARIABLE BIT RATE}

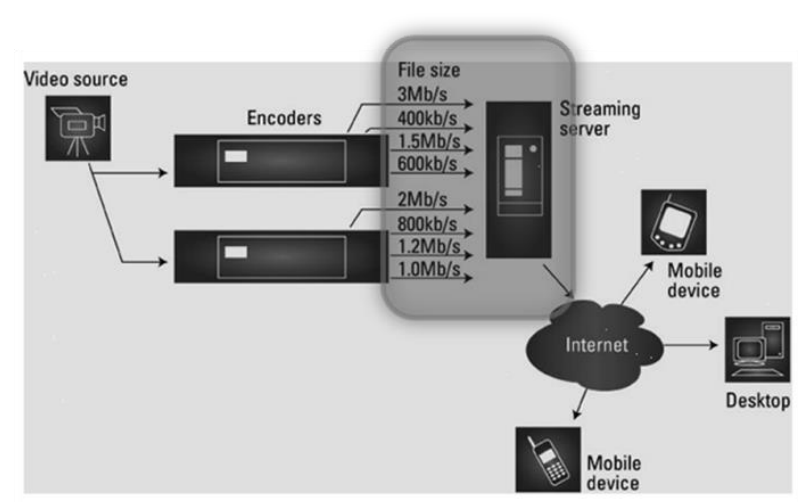

Figure 1. Process VBR in MANET

Variable Bitrate (VBR) is a term used in telecommunications and computing that relates to the bitrate used in sound or video encoding. As opposed to constant bitrate (CBR), VBR files vary the amount of output data per time segment. VBR allows a higher bitrate (and therefore more storage space) to be allocated for more complex segments of media files while less space is allocated to less complex segments. The average of these rates can be calculated to produce an average bitrate for the file.

MP3, WMA, Vorbis, and AAC audio files can optionally be encoded in VBR. Variable bit rate encoding is also commonly used on MPEG-2 video, MPEG-4 Part 2 video (Xvid, DivX, etc.), MPEG-4 Part 10/H.264 video, Theora, Dirac and other video compression formats. Additionally, the variable rate encoding is inherent in lossless compression schemes such as FLAC and Apple Lossless.

VBR produces a better quality-to-space ratio compared to a CBR file of the same data. The bits available are used more flexibly to encode the sound or video data more accurately, with fewer bits used in less demanding passages and more bits used in difficult-to-encode passages.

\section{MAP READING}

Our node by node classification faced with the task of establishing a route in a MANET, one's first thought might be to apply a position based routing algorithm [3]. While this 
work can be used in small networks with slowly moving nodes, it is not efficient. When we receive information about the location is available offered by GPS, another option is to use geographic routing [6]. The algorithm's spontaneous appeal has won it status. The focus of the method is simply to forward frames in the direction of the goal. When this is not possible, a contingency plan is employed, often at a steep cost in traffic and delay, or the link is dropped. By mobility tracking we refer to the act of keeping track of a node's position in order to estimate where it will be in the future. In this simple case, which we study here, each node tracks only itself. This general case, each node tracks some other nodes, using a distributed algorithm. The research on mobility tracking in the context of wireless networks has usually focused on dead reckoning [8], only recently considering more sophisticated approaches involving machine learning. Greater attention has been paid to this issue by the robotics community [1] and the pervasive computing community [10].

\section{SCALABILITY ISSUES IN ROUTING MANET}

Routing in wireless networks, especially with a large number of nodes, requires a different approach from those in fixed networks. The reason is that conventional routing algorithms attempt to minimize the number of bounds without concern for the overhead incurred by the direction-finding algorithm. This strategy is not successful in mobile ad hoc networks because the routing overhead constitutes a greater fraction of the overall traffic. Therefore, it imposes a fundamental limit on the scalability of wireless networks. Furthermore, different applications may demand optimizing different metrics like delay, dependability, and network lifetime. The rapidly changing topology exacerbates the problem, as the location manager struggles to maintain accurate estimates of the nodes' location. This assumes that reducing the end-to-end delay is a priority. If not, then it is actually possible to increase the transport capacity by using diversity routing.

\section{ALGORITHM}

To the best of our acquaintance, the closest results are analyzing the impact of delay on throughout in. However, they assume that only one intermediate is forwarded through and that diversity routing and diversity coding are used. In contrast, because of VBR passing, we are interested in characterizing the energy savings by providing future location information, for following belongings:

- Machine learning: Mechanical classification coordination to avoid channel disputation - passagetracking

- Future is known only one's own.

- The future of one's neighbors is also known.

- $\quad$ To keep up connectivity by significant how much energy is absent: Energy-tracking by Machine Learning?

- To stay away from dead ends with geographic steering: Route Map-structure by Machine learning

Our goal is simply to make the best forwarding decision for geographic routing next bound in order to extend the lifetime of the network. We intend to determine the energy savings of a simulated with respect to the amount of future knowledge and maximum permissible delay. We consider the network lifetime and the end-to-end route power.

\section{A. Machine Learning}

Mechanical classification coordination to avoid channel disputation - passage-tracking. In this phase, we classify the traffic tracking by using cameras [7].

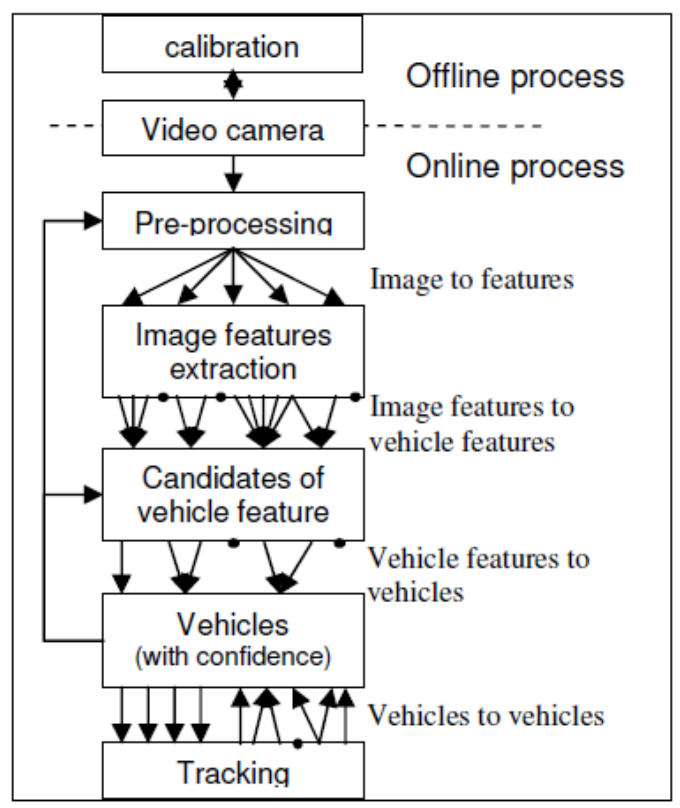

Figure 2. Vehicle Features Detection and Tracking.

It's a process of traffic tracking $b$ using cameras in the mode of machine learning system

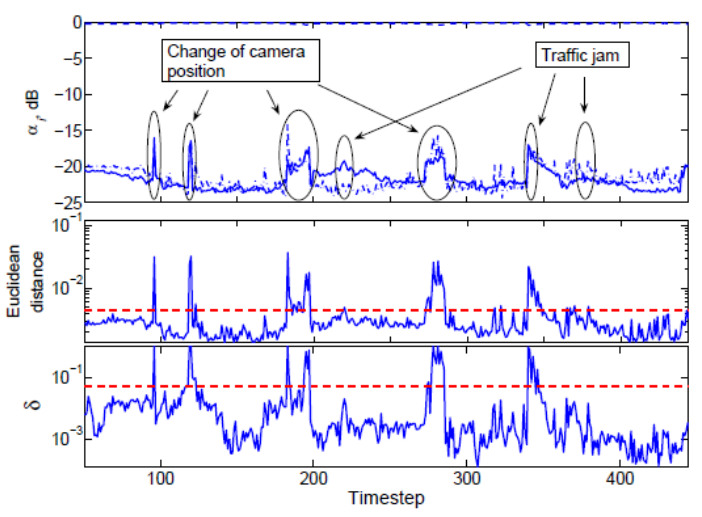

Figure 2.1. The plot examine the results

Traffic jam and time taken in real time traffic ad hoc network by using cameras. Top panel: Annotated plot of average wavelet coefficients in sub bands evolving through time. Approximation coefficients are shown by dashed line. Solid and dashed lines indicate transform levels 1 and 6 corresponding to highest and lowest frequency. Middle panel: OCNM using $\mathrm{K}^{\text {th }}$ nearest-neighbor distance, with dashed line indicating 90\% MVS threshold. Bottom panel: projection error_t with dashed line indicating lower threshold. Transports Quebec dataset [7]. 


\section{B. Route Map - Structure by machine learning}

We use the example in Figure. 3 to illustrate the basic ideas in node bound identification system. We assume that some nodes have already been selected as landmark nodes by the Node place range and each node knows its bound distance to all node location. In Fig. $1, \mathrm{~L}_{1}, \mathrm{~L}_{2}$ and $\mathrm{L}_{3}$ are three possible locations. Following a predefined order, the bound distance of a node to all the areas is combined into a vector, i.e. the node's bound reference identification number. For example, $\mathrm{L}_{2}$ 's bound number is 305 in Figure. 3, representing that $L_{2}$ is 3 bounds away from $\mathrm{L}_{1}, 0$ bound away from itself and 5 bounds away from $\mathrm{L}_{3}$.

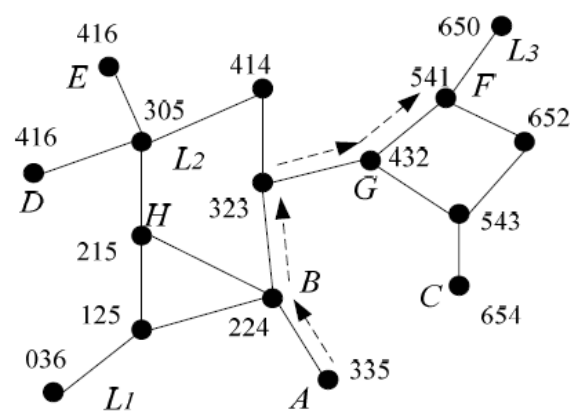

Figure 3. To stay away from dead ends with geographic steering: Route Mapstructure.

Figure. 3 Example of Bound number. A node N's Bound Number xyz means $\mathrm{N}$ is $\mathrm{x}, \mathrm{y}, \mathrm{z}$ bounds away from landmark $\mathrm{L}_{1}$, $\mathrm{L}_{2}$ and $\mathrm{L}_{3}$ respectively. Intuitively, the Bound Number can reflect the proximity of the network to some extent. Take two nodes $\mathrm{N}_{1}$ and $\mathrm{N}_{2}$ for example, we define the bound distance between $\mathrm{N}_{1}$ and $\mathrm{N}_{2}$ as $\mathrm{L}_{\mathrm{h}}$. Assume there are m landmark nodes, and the Bound Number of $\mathrm{N}_{1}$ is

$\mathrm{H}_{\mathrm{K}}{ }^{(1)}\left(\mathrm{H}_{1}{ }^{(1)}, \mathrm{H}_{2}{ }^{(1)}, \ldots \mathrm{H}_{\mathrm{m}}{ }^{(1)}\right)$, bound number of $\mathrm{N}_{2}$ is $\mathrm{H}_{\mathrm{K}}{ }^{(2)}\left(\mathrm{H}_{1}{ }^{(2)}\right.$ $\left., \mathrm{H}_{2}{ }^{(2)}, \ldots \mathrm{H}_{\mathrm{m}}{ }^{(2)}\right)$, the following triangulation inequality holds:

$\operatorname{Max}_{\mathrm{K}}\left(\left|\mathrm{H}_{\mathrm{K}}^{(1)}-\mathrm{H}_{\mathrm{k}}^{(2)}\right|\right) \leq \mathrm{L}_{\mathrm{h}} \leq \operatorname{Min}_{\mathrm{K}}\left(\mathrm{H}_{\mathrm{k}}^{(1)}+\mathrm{H}_{\mathrm{k}}^{(2)}\right)(1)$.

according to the grapevine, for each $k$ from 1 to $m, L_{h}$ is no more than the sum of $\mathrm{H}_{\mathrm{K}}{ }^{(1)}$ and $\mathrm{H}_{\mathrm{K}}{ }^{(2)}$, since there exists a path from $N_{l}$ to $N_{2}$ via landmark $k$ and the bound count of this path is $\mathrm{H}_{\mathrm{k}}{ }^{(1)}+\mathrm{H}_{\mathrm{k}}{ }^{(2)}$. For the left part of the inequality, without losing the generality, we assume $\mathrm{H}_{\mathrm{k}}{ }^{(1)}$ is no more than $\mathrm{H}_{\mathrm{k}}{ }^{(2)}$. $\mathrm{H}_{\mathrm{k}}{ }^{(2)}$ is no more than the sum of $L_{h}$ and $\mathrm{H}_{\mathrm{k}}{ }^{(1)}$, because there is a path from landmark $k$ to $N_{2}$ via node $N_{l}$ and $\mathrm{H}_{\mathrm{k}}{ }^{(2)}$ is the shortest bound distance from landmark $k$ to $N 2$. These inequalities yield a lower bound $L$ and an upper bound $U$ of $L_{h}$. More landmark nodes can make the lower and upper bounds. The number of landmarks needed in reality will be a constant which is determined by the precision requirement other than number of nodes in the network. By using above algorithm process, we effectively route make the best forwarding decision for geographic routing in order to extend the lifetime of the network. We intend to determine the energy savings of a simulated MANET with respect to the mount of future knowledge and maximum permissible delay.

\section{MAP READING}

Wireless networks of sensors are likely to be widely deployed in the near future because they greatly extend our ability to monitor and control the physical environment from remote locations and improve our accuracy of information obtained via collaboration among sensor nodes and online information processing at those nodes. Our goal is simply to make the best forwarding decision for geographic routing next bound in order to extend the lifetime of the network. We intend to determine the energy savings of a simulated with respect to the amount of future knowledge and maximum permissible delay. We regard as the network lifetime and the end-to-end route power. In further research is to implement this functionality fully in machine learning system.

\section{ACKNOWLEDGMENT}

Our thanks to the experts who have contributed towards development of the work.

\section{REFERENCES}

[1] Abdelsalam, W. and Ebrahim, Y., Managing Uncertainty:Modeling Users in Location-Tracking Applications, IEEE Pervasive Computing, vol. 3, no. 3, pp. 60-65,2004

[2] BENNEWITZ, M., BURGARD, W., CIELNIAK, G. AND THRUN, S., Learning Motion Patterns of People for Compliant Robot Motion, The International Journal of Robotics Research, vol. 24, no. 1, pp. 31-48, Jan. 2005 DOI: 10.1177/0278364904048962.

[3] GUPTA, P. AND KUMAR, P. R., The Capacity of Wireless Networks, IEEE Transactions on Information Theory, vol. 46, no. 2, pp. 388-404, Mar. 2000 DOI:10.1109/18.825799.

[4] C. Scott and R. Nowak, Learning minimum volume sets, J. Machine Learning Research (JMLR), vol. 7, pp. 665-704, Apr. 2006.

[5] KWOK, C., FOX, D. AND MEIL`A, M., Real-Time Particle Filters, Proceedings of the IEEE, vol. 92, no. 3, pp. 469-484, Mar. 2004 DOI : 10.1109 / JPROC.2003.823144.

[6] STOJMENOVIC, I., Position-Based Routing Algorithms in Ad Hoc Networks, IEEE Com

[7] Machine Learning Approaches to Network Anomaly Detection, Tarem Ahmed, Boris Oreshkin and Mark Coates Department of Electrical and Computer Engineering McGill University Montreal, QC, Canada

[8] KUMAR, V. AND DAS, S. R., Performance of Dead Reckoning-Based Location Service for Mobile Ad Hoc Networks, Wiley Wireless Communications and Mobile Computing, vol. 4, no. 2, pp. 189-202, Mar. 2004 DOI:10.1002/wcm.163.

[9] L. Ruan, H. Du, X. Jia, W. Wu, Y. Li, and K.-I. Ko. A greedy approximation for minimum connected dominating sets. Theoretical Computer Science, 329(1-3):325-330, 2004 unications Magazine, vol. 40, no. 7, pp. 128-134, Jul. 2002 DOI:10.1109/MCOM.2002.1018018.

[10] M. Chatterjee, S. Das and D. Turgut. WCA: A Weighted Cluster- ing Algorithm for Mobile Ad Hoc Networks. Journal of Cluster Computing (Special Issue on Mobile Ad hoc Networks), Vol. 5, No. 2, April 2002, 193-204.

[11] Anindya Iqbal, Nafees Ahmed, and Md. Mostofa Akbar. Directional antenna based connected dominating set construction for energy efficient broadcasting in wireless ad hoc network Computer and Electrical Engineering, International Conference on, 0:839-843,2008.

V.Saravanan received M.Sc(CS)., from Bharathiar University in 1999, completed M.Phil., from Manonmaniam Sundaranar University in 2002. Received MCA., from Periyar University in 2011. Currently working in Asst. Professor in Dept. Of Computer Applications, Hindusthan College of arts and science, Coimbatore. His research area is Network security in mobile networking.

Dr. C.Chandrasekar is an Associate Professor in Dept. Of Computer Science, Periyar University, salem. He has done MCA., Ph.D., He is been in the Teaching and Research field for more than 15 years. His Research area is Mobile and Wireless computing. He has worked and published more than 40 research articles. 